\title{
RELATO DE EXPERIÊNCIA SOBRE O PROJETO DE DISTORÇÃO HARMÔNICA TOTAL: METODOLOGIA ATIVA EM ELETRÔNICA ANALÓGICA NO CONTEXTO DE PANDEMIA DA COVID-19
}

DOI: 10.37702/2175-957X.COBENGE.2021.3648

Wendler Luis Nogueira Matos - wendleerluis@gmail.com

Universidade Federal do Pará

Rua comandante Francisco de Assis 2793

68743-100 - Castanhal - PA

Roberto Menezes Rodrigues - rmr@ufpa.br

UFPA

rua augusto correa $\mathrm{s} / \mathrm{n}$

66075-110 - Belém - PA

Thiago Carvalho dos Santos - thiago.csantos@itec.ufpa.br Universidade Federal do Pará

Travessa Santos Dumont 1586

68440-000 - Abaetetuba - PA

Kalil Brito Souza de Almeida - kalil.almeida@itec.ufpa.br

Universidade Federal do Pará

Travessa Vileta 3400

66095-346 - Belém - PA

Pablo Henrique Lisboa Caldas - pablohlcaldas@gmail.com

Universidade Federal do Pará

Avenida Eng. Fernando Guilhon $12 \mathrm{C}$

66030-365 - Belém - PA

Resumo: Devido ao grande número de disciplinas abordadas, os alunos dos cursos das áreas das ciências aplicadas, como a engenharia elétrica, costumam ter dificuldade em assimilar os tópicos mais importantes. Entre os recursos facilitadores estão as aulas laboratoriais, onde o aluno pode confrontar e vivenciar os conceitos teóricos na prática. Atualmente, as aulas estão sendo realizadas remotamente devido à pandemia da COVID-19. Isso impõe um grande desafio à 
qualidade da formação dos alunos. Uma alternativa viável e principalmente adequada à realidade atual é a utilização de simuladores computacionais. Em disciplinas como a eletrônica analógica, os simuladores podem ser utilizados como ferramenta de metodologia ativa, possibilitando a consolidação do conhecimento de forma prática e bem estruturada que estimula a criatividade dos alunos, como se estivessem em um laboratório real. $O$ presente trabalho tem como objetivo relatar a experiência na execução de um projeto educacional envolvendo o conceito de Total Harmonic Distortion (THD) - Distorção Harmônica Total em circuitos eletrônicos, durante a disciplina de eletrônica analógica 2, do curso de Engenharia Elétrica da Universidade Federal do Pará (UFPA). Em particular, busca avaliar a importância da utilização de softwares de simulação como ferramenta de metodologia ativa em tempos de contato social restrito, como é o caso atualmente vivenciado.

Palavras-chave: Amplificadores, Distorção Harmônica Total, Eletrônica Analógica, Filtros, Metodologia de Ensino 


\section{RELATO DE EXPERIÊNCIA SOBRE O PROJETO DE DISTORÇÃO HARMÔNICA TOTAL: METODOLOGIA ATIVA EM ELETRÔNICA ANALÓGICA NO CONTEXTO DE PANDEMIA DA COVID-19}

\section{INTRODUÇÃO}

Os cursos de engenharia demandam uma série de conhecimentos em softwares, sejam de construção de circuitos, sistemas de controle, sistemas de energia dentre outros. A utilização desses programas é de fundamental importância para que se simule situações reais com segurança, já que na construção real de um protótipo podem ocorrer diversos erros que podem comprometer a integridade funcional dos equipamentos.

A disciplina de eletrônica analógica é fundamentalmente prática, pois estuda sinais e dispositivos analógicos, como circuitos envolvendo capacitores, indutores e diodos. Em um contexto de pandemia global da Corona Virus Disease (COVID-19) - Doença do Coronavírus), muitas instituições tiveram que adotar o ensino remoto emergencial como solução para a não suspensão total das aulas. Com isso, é necessário aproveitar ao máximo as tecnologias digitais para mediar a aprendizagem remota (FERREIRA; BRANCHI; SUGAHARA, 2020). Quanto a disciplina em questão, o ensino-aprendizagem nesse contexto é muito prejudicado, devido a necessidade de se abstrair muitos conceitos que poderiam ser abordados de modo prático com uma maior interação com o professor em sala de aula. Por isso, é necessário adotar metodologias e estratégias que utilizem softwares para que o aluno se sinta o mais próximo possível de uma situação real, observando erros nos projetos e os resultados esperados. É possível afirmar então que, com a ocorrência da pandemia, a praticidade e abrangência dos simuladores se adequam às necessidades do ensino remoto.

Segundo Freire (1996), o aprendizado dos alunos só ocorre quando existe participação ativa nos processos. Logo, é necessário construir experiências práticas de problemas reais que permitam que os estudantes contextualizem a situação e estimulem seus pensamentos para solucionar o problema em questão. Assim, com ausência de aulas de laboratório entende-se que a única maneira dos alunos terem participação ativa é através de metodologias de ensino que façam uso de softwares para buscar aproximar ao máximo os conceitos vistos na parte teórica com o que possivelmente ocorreria na prática dentro de um laboratório, pois dessa forma o aluno irá associar as coisas de modo melhor, com mais chances de a teoria ser lembrada futuramente.

Em sala de aula normalmente tem-se aulas expositivas, através de lousas, livros e apresentações em slide, pois é mais prático ao professor (SILVA; MORAES; CUNHA, 2011). Porém, com a evolução do ensino-aprendizagem, foram criadas diversas metodologias para melhorar a absorção de conhecimento pelos alunos. Uma delas é a Project Based Learning (PBL) - Aprendizagem Baseada em Projetos; que defende a necessidade de se utilizar problemas da vida real para facilitar/motivar a aprendizagem dos conceitos de uma dada disciplina, mostrando situações que os alunos possam se deparar nas suas futuras atuações profissionais (RIBEIRO, 2008). Esse método não é contra as aulas expositivas, apenas critica aquelas que se limitam em apresentar apenas fundamentos teóricos técnico-científicos previstos na componente curricular.

Uma situação real dentro do curso de engenharia elétrica é trabalhar com sinais elétricos, sejam digitais ou analógicos. Um tópico importante dentro da disciplina de eletrônica analógica 2 é a distorção não-linear harmônica de sinais. Essa área estuda a presença de componentes harmônicas indesejadas no sinal (BOYLESTAD; NASHELSKY, 
2013). Por exemplo, se o objetivo é obter na saída de um circuito uma forma de onda senoidal com frequência fundamental de $60 \mathrm{~Hz}$, mas ao visualizar-se a saída seja em um osciloscópio seja em um software de simulação detectou-se a presença das harmônicas 2 e 3, significa que o sinal possui componentes nas frequências de $120 \mathrm{~Hz}$ e $180 \mathrm{~Hz}$ (além da fundamental de $60 \mathrm{~Hz}$ ). Como não se queria isso, existiu uma distorção, quantificada pelo valor de Total Harmonic Distortion (THD) - Distorção Harmônica Total, que varia de 0\% (distorção nula) até mais de 100\%, revelando grande distorção.

O objetivo do trabalho é relatar a experiência do grupo na execução do projeto eletrônico envolvendo o conceito de THD, da disciplina eletrônica analógica 2, a partir de uma metodologia ativa de uso de software de simulação.

O restante do trabalho está organizado como segue. Na seção 2, aborda-se a metodologia do projeto bem como as etapas de execução. Na seção 3, apresenta-se a experiência que a equipe teve no desenvolvimento do projeto. Por fim, as conclusões do trabalho são apresentadas na seção 4.

\section{METODOLOGIA}

Nessa seção é descrita a elaboração do projeto sobre THD pelos discentes do curso de engenharia elétrica da Universidade Federal do Pará (UFPA), referente a um projeto de ensino da disciplina de eletrônica analógica 2. O mesmo foi realizado na Oferta $B$ do Ensino Remoto Emergencial (ERE), período que correspondeu entre 10/12/2020 e 26/02/2021. O projeto sobre THD foi motivado pelo contexto de pandemia da COVID-19, sendo então uma metodologia ativa de uso de programa computacional para auxiliar no aprendizado, relacionando a teoria dos sinais analógicos com o desenvolvimento prático de simulação em um software (escolheu-se utilizar o LTspice). As especificações do projeto, definição de THD e discussões a respeito de cada bloco funcional serão apresentadas a seguir.

\subsection{Especificações do projeto THD}

O objetivo era projetar um sistema eletrônico que gerasse uma forma de onda senoidal a mais pura possível (apenas com frequência fundamental) ao final do processo. O circuito deveria ser composto de 4 estágios/blocos funcionais, sendo: Gerador de onda quadrada com Circuito Integrado $(\mathrm{Cl}) 555$, filtro, multiplicador de frequência e amplificador com TBJ (Transistor Bipolar de Junção). A Figura 1 mostra o diagrama de blocos do sistema.

Figura 1 - Diagrama de blocos do sistema a ser construído.

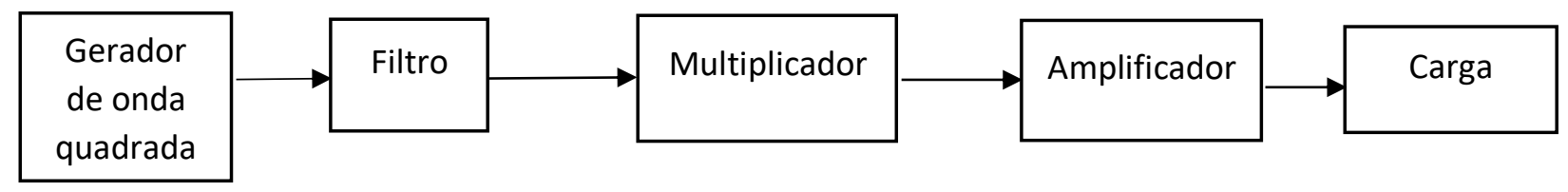

Fonte: Autores.

O Gerador de onda quadrada com Cl 555 representa um oscilador local. É projetado a partir de um Circuito Integrado 555 no modo astável. A frequência de onda pôde ser escolhida, dentro da faixa de $3 \mathrm{KHz}$ a $15 \mathrm{KHz}$. Além disso, o ciclo de trabalho (tempo em nível alto dividido pelo tempo total do ciclo) deveria ser de $50 \%$, com possível variação de mais ou menos $5 \%$. O filtro passa-faixa é responsável por filtrar apenas a componente harmônica desejada. A estrutura do filtro pôde ser escolhida, com a limitação de ordem do filtro até 3 . O objetivo nessa etapa é ter um filtro passa-faixa centrado na frequência da $5^{\mathrm{a}}$ harmônica do sinal de entrada (onda quadrada que sai do Cl 555). Por 
exemplo, se a frequência fundamental da onda quadrada do $\mathrm{Cl} 555$ fosse de $1 \mathrm{KHz}$, se deveria ter na saída do filtro a frequência de centro de $5 \mathrm{KHz}$.

No multiplicador de frequência deseja-se que o sinal de saída possua frequência fundamental 2 vezes a da entrada (sinal que sai do filtro). Por exemplo, se na saída do filtro tem-se frequência de centro de $5 \mathrm{KHz}$, na saída do multiplicador se deseja $10 \mathrm{KHz}$. Com relação à topologia, o dobrador de frequência deve ser composto por um bloco gerador de harmônicas seguido de um filtro sintonizado no dobro da frequência original. $\mathrm{O}$ bloco gerador de harmônicas deveria ser construído com diodo (s).

O amplificador deve ser do tipo Fonte de Tensão Controlada por Tensão (FTCT) e é responsável por compensar as perdas (de amplitude) ocorridas nos estágios anteriores. O elemento ativo desse bloco será o amplificador com Transistor Bipolar de Junção (TBJ). A configuração poderia ser escolhia (emissor comum, base comum ou coletor comum), bem como a escolha do esquema de polarização. O ganho de tensão requerido era tal que a carga, que simbolizava um equipamento hipotético que iria receber o sinal senoidal de saída dos 4 blocos e cuja resistência poderia ser escolhida (entre $1 \mathrm{~K} \Omega$ a $5 \mathrm{~K} \Omega$ ) percebesse uma tensão pico a pico entre $15 \mathrm{~V}$ e $20 \mathrm{~V}$. Além disso, caso fosse conveniente, poderia ser usado múltiplos estágios de amplificação.

Finalmente, uma vez que o circuito seja projetado segundo as especificações, deve-se avaliar o nível de distorção gerado pelo mesmo através da métrica THD. Mais especificamente, o valor de THD na saída do multiplicador de frequência, colocando esse bloco isolado, ou seja, com uma fonte senoidal ideal (de amplitude aproximadamente a que entrava no multiplicador no circuito geral); valor de THD na saída do amplificador, também colocando esse bloco isolado, ou seja, com uma fonte senoidal ideal (de amplitude aproximadamente a que entrava no amplificador no circuito geral); e valor de THD para o circuito geral, com todos os blocos em sequência. Tais valores de THD deveriam ser calculados limitando-se às 10 primeiras harmônicas. Todos os valores deveriam ser menores que $10 \%$.

\subsection{Distorção Harmônica Total (THD)}

Distorção é toda alteração da forma de onda de um sinal, em relação a uma forma de referência. Isso pode ocorrer devido a vários fenômenos, como por exemplo a partir da resposta não-linear que alguns componentes ativos apresentam. A Distorção Harmônica Total (THD) é uma das métricas mais populares na avaliação do grau de distorção nãolinear que um sistema gera em sua saída, tendo como estímulo uma senóide. Por nãolinear, entende-se a geração de componentes harmônicas que não estavam presentes no sinal original. Assim, a THD busca quantificar a energia encontrada nas harmônicas em comparação com a da fundamental (sinal original). Isso significa que para uma onda senoidal ideal, a THD é nula, pois nesta há somente uma componente de frequência que é a fundamental. A THD é importante em várias áreas da engenharia, como em sistemas de energia elétrica, nos quais um valor baixo representa um fator de potência mais elevado (evidenciando menos harmônicas devido a cargas reativas), e maior eficiência dos sistemas como um todo. Em sistemas de comunicação, quanto menor o valor de THD, menos interferência há na transmissão de um sinal, pois menos harmônicas interferiram na comunicação em canais vizinhos.

O cálculo da distorção harmônica total é dado através da Equação (1), onde $V_{p 1}$ é o valor de pico da tensão na frequência fundamental e os índices subsequentes $p_{1}, p_{2}$ até $p_{n}$ se referem aos valores de pico de tensão das harmônicas. 


$$
T H D=\frac{\sqrt{V_{p 1}^{2}+V_{p 2}^{2}+V_{p 3}^{2}+V_{p 4}^{2}+V_{p 5}^{2}+\cdots+V_{n}^{2}}}{V_{p 1}}
$$

\subsection{Bloco 01: Gerador de onda quadrada com Circuito Integrado 555}

O Circuito Integrado (Cl) 555 é muito utilizado em diversos projetos de eletrônica que demandam de temporizações, oscilações ou gerações de ondas quadradas, comumente para acionar dispositivos digitais. Possui algumas maneiras diferentes de interligação dos seus 8 pinos e dependendo da configuração que se utiliza o modo de operação pode variar. Existem três modos de operação: monoestável, biestável e astável. Segundo Braga (2016), o dispositivo possui tensão de operação que pode variar de 5 a 18 $\mathrm{V}$, fornecendo ou drenando correntes de até $200 \mathrm{~mA}$.

Os três modos de operação são classificados como multivibradores, sendo circuitos que apresentam dois estados definidos, ou saídas possíveis, sendo nível alto e nível baixo. Essa mudança de estado pode ser espontânea ou por meio de um pulso de comando chamado disparo. O circuito apresenta o modo estável (monoestável) quando necessita de um disparo para mudar de estado. Modo quase estável (biestável) quando retorna a um estado de origem decorrido um intervalo de tempo. Modo instável (astável) quando não mantém nenhum estado, alternando constantemente (LIMA JUNIOR, 2009).

No projeto aqui apresentado, configurou-se o $\mathrm{Cl} 555$ para operar como multivibrador astável ou oscilador. O componente produzirá constantemente pulsos de ondas quadradas que variam de $0 \mathrm{~V}$ a um nível chamado 'Vcc' a partir do carregamento $\mathrm{e}$ descarregamento de capacitores. Além disso, o modo astável permite alterar o tempo quando o capacitor carrega e descarrega, bastando dimensionar as resistências presentes nessa configuração. Primeiramente, selecionou-se a alimentação desse dispositivo como sendo $12 \mathrm{~V}$, e uma frequência de operação de $\mathrm{f}=10 \mathrm{KHz}$. Como foi requerido um ciclo de trabalho de $50 \%$ é necessário que $R_{2}$ seja aproximadamente 10 vezes maior que $\mathrm{R}_{1}$. Logo, escolheu-se $R_{1}=1 \mathrm{k} \Omega$ e consequentemente $R_{2}=10 \mathrm{k} \Omega$. Da Equação (2) encontrou-se $C=6,85 n F$ e da Equação (3) obteve-se um ciclo de $52 \%$.

$$
\begin{gathered}
C_{1}=\frac{1,44}{f\left(R_{1}+2 R_{2}\right)} \\
\frac{T_{H}}{T}=\left(\frac{R_{1}+R_{2}}{R_{1}+2 R_{2}}\right)
\end{gathered}
$$

A Figura 2 mostra como ficou o bloco 01 na sua configuração final modo astável, no software LTspice.

Figura 2. Bloco funcional $\mathrm{Cl} 555$. 


\subsection{Bloco 02: Filtro analógico}

Um filtro passivo passa faixa ou passa banda é aquele em que ocorre a passagem de sinais em uma determinada faixa de frequência. Ou seja, ele irá atenuar sinais que estejam abaixo ou acima de uma frequência específica. As delimitações dessa faixa são denominadas de frequência de corte inferior e frequência de corte superior.

O filtro delimita essa banda de passagem a partir de associações entre resistores, indutores e capacitores. Para altas frequências a maior parte da tensão de entrada estará sobre o indutor, que impede a passagem do sinal pois se comporta como um circuitoaberto. Já para baixas frequências a maior parte da tensão de entrada estará sobre o capacitor, que impede a passagem do sinal pois se comporta como um circuito-aberto. Para frequências intermediárias, próximas a de ressonância (central), o capacitor e o indutor se comportam como um curto-circuito. Portanto, o circuito permitirá passar sinais dentro desta faixa (MUSSOI, 2004). A ressonância é quando as reatâncias indutivas e capacitivas são iguais, apresentando um fator de potência unitário, e a frequência que provoca essa situação é chamada de frequência de ressonância (BERNARDO, 2021).

Para o projeto do filtro passa-faixa, especificou-se uma largura de banda que se inicia com $-3 d B$ de atenuação nas frequências $f_{1}=45 \mathrm{kHz}$ e $f_{2}=55 \mathrm{kHz}$, e termina com $-20 \mathrm{~dB}$ em $40 \mathrm{kHz}$ e $60 \mathrm{kHz}$. Lembrando que como a onda quadrada que sai do $\mathrm{Cl} 555$ possui $f=10 \mathrm{KHz}$, esse bloco precisa filtrar a harmônica $5(50 \mathrm{KHz})$. Para os cálculos dos parâmetros de capacitância e indutância foi considerado o valor de resistência $R_{10}=$ $70 \Omega$ para as resistências da fonte e da impedância de carga (embora como resultado final tenha-se alterado a resistência da fonte para $R_{9}=10 \Omega$, pois precisou-se dar mais tensão na saída do filtro) e também a arquitetura de Butterworth - que será descrita a partir de agora. A Equação (4) confirma que para as frequências escolhidas tem-se $f_{0}=50 \mathrm{kHz}$.

$$
f_{0}=\sqrt{f_{1} f_{2}}
$$

Para se determinar as frequências de banda de parada é necessário supor primeiro $f_{a}=40 \mathrm{kHz}$ e depois $f_{b}=60 \mathrm{kHz}$. A Equação (5) mostra para $f_{a}=40 \mathrm{kHz}$, na qual se encontrou $f_{b}=62,5 \mathrm{kHz}$ e relação $f_{b}-f_{a}=22,5 \mathrm{kHz}$. Já a Equação (6) mostra para $f_{b}=$ $60 \mathrm{kHz}$, na qual se encontrou $f_{a}=41,67 \mathrm{kHz}$ e relação $f_{b}-f_{a}=18,33 \mathrm{kHz}$. Deve-se escolher as frequências que resultaram no menor valor de $f_{b}-f_{a}$, para se calcular o fator de inclinação mais adequado. A Equação (7) representa o cálculo desse fator, na qual a banda foi de $18,33 \mathrm{kHz}$ e a largura para $-3 \mathrm{~dB}$ foi $10 \mathrm{k} \mathrm{Hz}$, que resultou em $A_{s}=1,83$.

$$
\begin{gathered}
f_{b}=\frac{f_{0}^{2}}{f_{a}} \\
f_{a}=\frac{f_{0}^{2}}{f_{b}} \\
A_{s}=\frac{\text { banda de parada da largura de banda }}{3 d B \text { de largura de banda }}
\end{gathered}
$$

Nesse momento, analisou-se as curvas de Butterworth, com o objetivo de encontrar qual ordem do filtro resultaria em $-20 \mathrm{~dB}$ de atenuação com velocidade de 1,88 $\mathrm{rad} / \mathrm{s}$. Na curva isso foi obtido quando $n=3$ (SCHERZ, MONK, 2013), representando um filtro passafaixa de terceira ordem. O próximo passo foi calcular os parâmetros de capacitância e impedância do filtro passa-faixa normalizado. Considerando a resistência da carga $R_{10}=$ 
$70 \Omega, \Delta f=f_{1}-f_{2}=10 \mathrm{kHz}$ e utilizando a tabela de Butterworth (já sabendo que $\mathrm{n}=3$ ), foi possível obter: $C_{1 \text { (tabela) }}=1 \mathrm{~F}, L_{2 \text { (tabela) }}=2 \mathrm{H}$ e $C_{3(\text { tabela })}=1 \mathrm{~F}$. Com isso encontrou-se $C_{2}=0,21 \mu F ; L_{2}=2,24 m H$ e $C_{4}=0,21 \mu F$ a partir das Equações (8) (9) e (10), respectivamente.

$$
\begin{gathered}
C_{2}=\frac{C_{1(\text { tabela })}}{2 \pi \Delta f R_{L}} \\
L_{2}=\frac{L_{2(\text { tabela })} R_{L}}{2 \pi \Delta f} \\
C_{4}=\frac{C_{3(\text { tabela })}}{2 \pi \Delta f R_{L}}
\end{gathered}
$$

Para o filtro passa-baixa ressoar com a frequência fundamental $f_{0}=50 \mathrm{KHz}$, deve-se adicionar um capacitor em série com o indutor $L_{2}$ e indutores em paralelo com os capacitores $C_{2}$ e $C_{4}$. As Equações (11) (12) e (13) permitiram calcular, respectivamente, $L_{1}=47 \mu \mathrm{H}$ que está em paralelo com $C_{2}, C_{3}=4,28 \mathrm{nF}$ que está em série com $L_{2}$ e $L_{3}=$ $47 \mu \mathrm{H}$ que está em paralelo com $C_{4}$. A Figura 3 mostra o resultado final do filtro.

$$
\begin{aligned}
& L_{1}=\frac{1}{\left(2 \pi f_{0}\right)^{2} C_{2}} \\
& C_{3}=\frac{1}{\left(2 \pi f_{0}\right)^{2} L_{2}} \\
& L_{3}=\frac{1}{\left(2 \pi f_{0}\right)^{2} C_{4}}
\end{aligned}
$$

Figura 3 - Bloco funcional filtro passa-faixa.

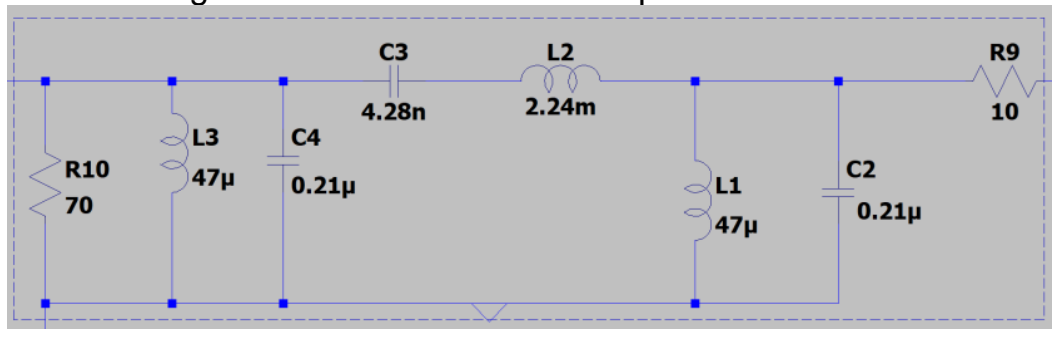

Fonte: Autores.

\subsection{Bloco 03: Multiplicador de frequência}

Após a etapa de filtragem da onda quadrada, tem-se na saída uma onda senoidal de frequência $50 \mathrm{KHz}$. O multiplicador de frequência é responsável por dobrar a frequência para $100 \mathrm{KHz}$. Segundo as especificações de projeto, para este bloco, foi utilizado somente componentes passivos: diodo, capacitores e indutores, tornando este bloco puramente passivo, assim como o filtro analógico. Houveram complicações no projeto desse bloco devido à dificuldade em se encontrar na literatura bibliográfica essa topologia, sendo a mais utilizada a versão que utiliza indutores de tomada central. Para a 
descrição deste circuito pode-se dividir em duas partes: diodo semicondutor e ressonadores.

De forma básica, o princípio do funcionamento de um diodo semicondutor é conduzir a corrente elétrica em apenas um sentido. É um elemento não-linear que distorce uma onda senoidal e para o seguinte projeto foi escolhido o diodo comercial $1 \mathrm{~N} 4148$, pois este dispositivo pode operar em altas frequências e manter a sua nãolinearidade. Além disso, ele está disponível na biblioteca de componentes do software LTspice. Quando há distorção de uma onda senoidal, ocorre a geração de componentes harmônicas de frequências. Então, o diodo do multiplicador de frequência tem como princípio fundamental distorcer uma onda e gerar frequências harmônicas que são múltiplas da frequência fundamental. Com isso, obtém-se componentes múltiplas de 50 $\mathrm{KHz}$ (saída do filtro e entrada desse bloco) e objetiva-se filtrar a harmônica 2 (de 100 $\mathrm{KHz}$ ) para cumprir a especificação do projeto; sendo esse bloco um duplicador de frequência.

Os ressonadores apresentam um papel fundamental para a multiplicação da frequência, uma vez que, após a distorção do sinal é necessário reverter esse efeito e obter uma onda senoidal ideal na saída do multiplicador. Eles são compostos por um capacitor em paralelo com um indutor e tem características que diferem de acordo com a frequência em que operam. Os ressonadores LC nesse projeto serão responsáveis por curto-circuitar o diodo nas harmônicas indesejadas, desacoplando a entrada da saída, e colocar o diodo em paralelo com a entrada na frequência fundamental e em paralelo com a saída na segunda harmônica (ROSU), fazendo assim filtragem da harmônica de 100 $\mathrm{KHz}$ que se deseja obter na saída e que foi gerada pela distorção da onda senoidal de entrada através do diodo. Através dos conceitos da teoria de circuitos, têm-se a Equação (14), que torna possível calcular a indutância, onde $\omega=2 \pi f, C$ é a capacitância, $L$ é a indutância e $f$ é a frequência de ressonância. Para ter a indutância escolheu-se os capacitor $C_{5}$ e $C_{6}$ de $50 \mathrm{nF}$ como referência e a frequência desejada pro trabalho é 100 $\mathrm{KHz}$; obteve-se $L_{4}=L_{5}=50,66 \mu \mathrm{H}$. A forma final desse bloco é mostrada na Figura 4 .

$$
f=\frac{1}{2 \pi \sqrt{L C}}
$$

Figura 4. Bloco funcional do multiplicador de frequência.

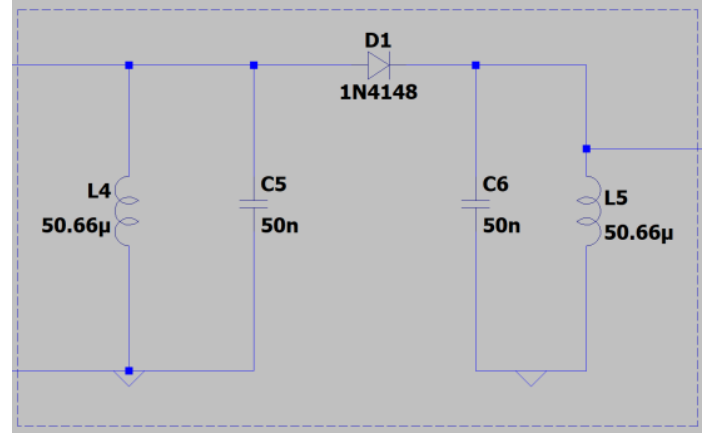

Fonte: Autores.

\subsection{Bloco 04: Amplificador com TBJ}

Quanto a essa etapa, que será responsável por amplificar o sinal de saída do multiplicador (dobrador de frequência), para uma tensão pico a pico final que precisava estar entre $15-20 \mathrm{~V}$ para carga sendo de $1-5 \mathrm{~K} \Omega$, a configuração final foi um estágio com amplificador emissor comum com polarização por divisor de tensão, que possui como característica gerar uma saída estável (SEDRA, 2015). Escolheu-se o transistor BC547B e $V_{c c}=36 \mathrm{~V}$. Para casamento de impedância, considerou-se $R_{C}=R_{L}$. Adotou-se 
resistência de carga de $2 \mathrm{~K} \Omega$. As especificações comumente adotadas na literatura são: $\mathrm{V}_{\mathrm{RE}}=0,1 \mathrm{Vcc}$; $\mathrm{V}_{\mathrm{CE}}=0,45 \mathrm{Vcc}$ e $\mathrm{V}_{\mathrm{RC}}=0,45 \mathrm{Vcc}$. Então, como $\mathrm{V}_{\mathrm{Cc}}=36 \mathrm{~V}$ : $\mathrm{V}_{\mathrm{RE}}=3,6 \mathrm{~V} ; \mathrm{V}_{\mathrm{CE}}=16,2$ $\mathrm{V}$ e $\mathrm{V}_{\mathrm{RC}}=16,2 \mathrm{~V}$. A Equação (15) permitiu encontrar a corrente no coletor como sendo $I_{c}=$ 8,1 $\mathrm{mA}$. Com essa corrente e analisando o datasheet do BC547B e especificações do LTspice, o valor do 'beta' do transistor foi $\beta=294$ e a Equação (16) permitiu encontrar a corrente na base $I_{b}=27,55 u A$. Sabendo que a corrente no emissor $I_{e}$ é igual à soma entre as duas anteriores, teve-se $I_{e}=8,13 \mathrm{~mA}$.

$$
\begin{gathered}
I_{C}=\frac{V_{R C}}{R_{C}} \\
I_{b}=\frac{I_{c}}{\beta}
\end{gathered}
$$

Sabendo disso, pôde-se encontrar o resistor do emissor através da Equação (17), encontrando $R_{E}=442,8 \Omega$. A Equação (18) permitiu calcular a resistência interna do emissor, sabendo que a tensão interna é de aproximadamente $26 \mathrm{mV}$. Obteve-se $r_{e}=$ $3,2 \Omega$.

$$
\begin{array}{r}
R_{E}=\frac{V_{E}}{I_{E}} \\
r_{e}=\frac{V_{t}}{I_{E}}
\end{array}
$$

O próximo passo foi encontrar a resistência $R_{4}$. Para isso adota-se comumente que $I_{R 4}$ é aproximadamente igual a $10 I_{b}$. Logo $I_{R 4}=0,275 \mathrm{~mA}$. Além disso, sabe-se, da teoria de amplificadores, que $V_{R 4}=V_{R E}+V_{B E}$, sendo $V_{B E}$ aproximadamente $0,7 \mathrm{~V}$. Com isso, 0 valor de $V_{R 4}$ foi de 4,3 V. Assim, a Equação (19) calcula a resistência $R_{4}$, obtendo 15,63 $\mathrm{K} \Omega$. A última resistência a ser encontrada é $R_{3}$. Como $V_{R 3}=V_{c c}-V_{R 4}=31,7 \mathrm{~V}$ e sabe-se que $I_{R 3}=I_{B}+I_{R 4}$, resultando em $I_{R 3}=0,3 \mathrm{~mA}$, a Equação (20) permite então calcular $R_{3}$, encontrando $105,7 \mathrm{~K} \Omega$. Com todos os valores, calcula-se o ganho do amplificador a partir da Equação (21), que foi de 2,23. Como é muito pequeno, utiliza-se o capacitor $C_{8}$ que curto-circuita $\circ R_{E}$, e quando se recalcula o ganho resulta em 311,45 (ganho máximo permitido). Porém, quando se usa um valor alto a forma de onda da saída fica distorcida, então a solução é controlar o ganho, dividindo o resistor $R_{E}$ em $R_{E 1}+R_{E 2}$. Empiricamente nas simulações, verificou-se que o ganho que seria adequado para o projeto era de 35 . A Equação (22) permite calcular $R_{E 1}$, obtendo 25,27 $\Omega$. O cálculo de $R_{E 2}$ é apenas $R_{E 2}=$ $R_{E}-R_{E 1}=417,53 \Omega$. Para os 3 capacitores $\left(\mathrm{C}_{7}, \mathrm{C}_{8}\right.$ e $\left.\mathrm{C}_{9}\right)$ se escolheu um valor padrão de 10 uF. A Figura 5 mostra esse bloco completo, já com a carga no final.

$$
\begin{gathered}
R_{4}=\frac{V_{R 4}}{I_{R 4}} \\
R_{3}=\frac{V_{R 3}}{I_{R 3}} \\
\Delta V=\frac{I_{c}\left(R_{c} \| R_{L}\right)}{I_{b}\left(R_{E}+r_{e}\right)(\beta+1)}
\end{gathered}
$$




$$
R_{E 1}=\frac{I_{c}\left(R_{c} \| R_{L}\right)}{I_{b} \cdot 35 \cdot(\beta+1)}-r_{e}
$$

Figura 5. Bloco funcional do amplificador com TBJ.

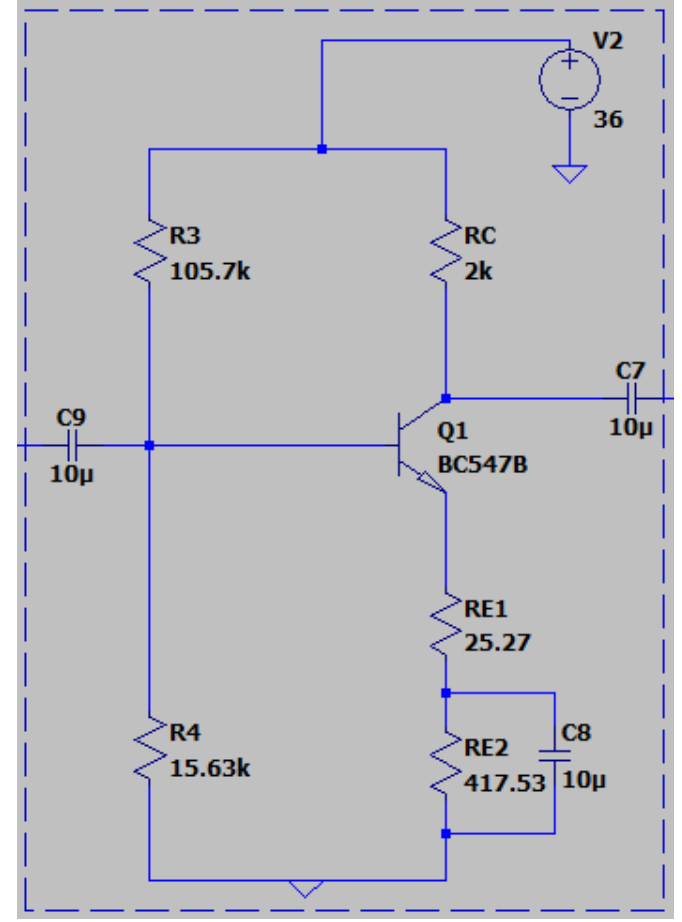

Fonte: Autores.

\section{$3 \quad$ RESULTADOS E DISCUSSÕES}

Para mensurar a THD pelo LTspice utilizou-se o comando ".four" e selecionou-se um limite de 10 harmônicas para o cálculo (especificação do trabalho), cujo valor final é exibido na janela "SPICE Error Log" em valor percentual. Para mensurar a THD na saída do multiplicador de frequência, retirou-se esse bloco do circuito geral e colocou-se em sua entrada uma senóide ideal de amplitude $0,5 \mathrm{~V}$ e frequência $50 \mathrm{KHz}$. A partir do uso da Equação (1) calculou-se o THD manualmente a partir da análise da Fast Fourier Transform (FFT) - Transformada Rápida de Fourier que o software realiza. O cálculo resultou em $0,75 \%$ e o valor do LTspice foi de $0,76 \%$.

Para mensurar a THD na saída do amplificador também se isolou esse bloco e foi colocado uma senóide ideal na sua entrada com amplitude de $280 \mathrm{mV}$ e frequência de $100 \mathrm{KHz}$. O valor obtido pelo LTspice foi de 6,74\% e o cálculo manual a partir da Equação (1) pela análise da FFT foi de 6,83\%. Por fim, a THD geral do circuito, com todos os blocos em sequência, foi obtida pelo software como sendo 5,61\% e o cálculo resultou em $5,55 \%$. Ou seja, todos os valores de THD ficaram abaixo dos $10 \%$ exigidos. Além disso, na saída obteve-se $16,6 \mathrm{~V}$ de tensão pico-a-pico para a carga escolhida de $2 \mathrm{~K} \Omega$. A Figura 6 mostra a saída na carga, com uma escala de tempo escolhida para melhor visualizar a senóide. A Figura 7 representa a FFT aplicada ao sinal de saída, mostrando a frequência fundamental de $100 \mathrm{KHz}$. 
Figura 6. Saída senoidal na carga de $2 \mathrm{~K} \Omega$.

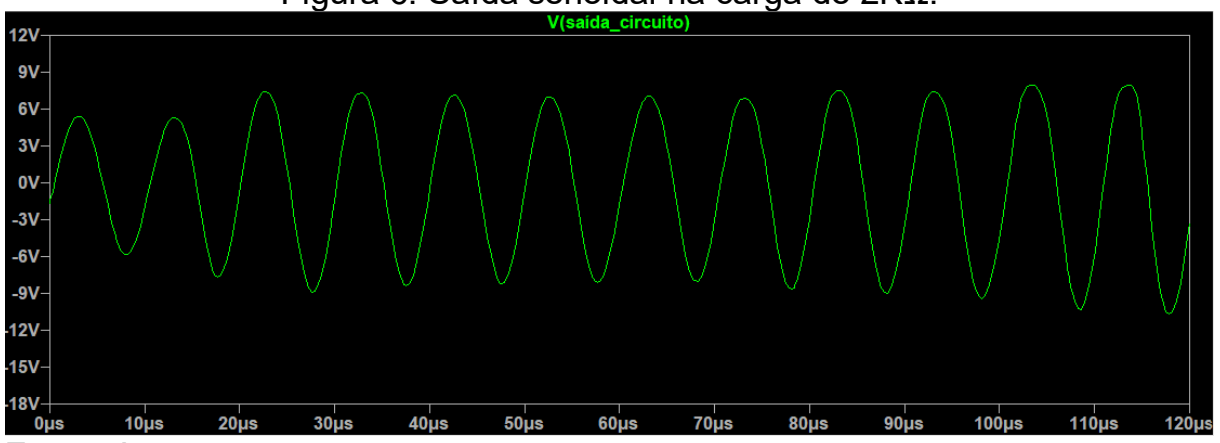

Fonte: Autores.

Figura 7. Transformada Rápida de Fourier aplicada ao sinal de saída na carga de $2 \mathrm{~K} \Omega$.

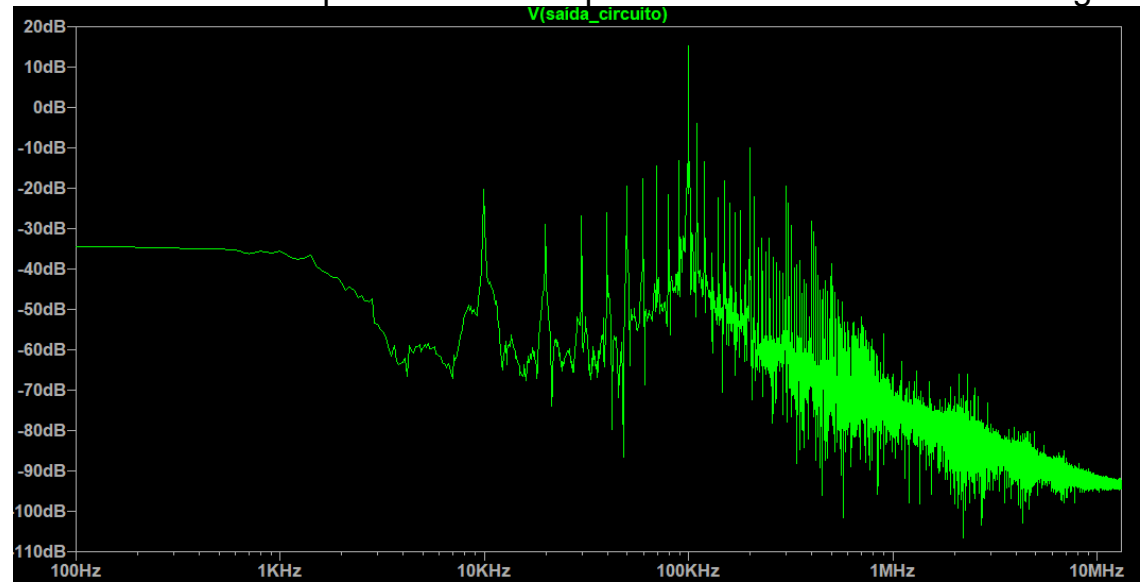

Fonte: Autores.

Quanto a experiência envolvida com o software de simulação LTspice, entende-se que foi a metodologia ativa mais adequada possível para reduzir a lacuna de aprendizagem consequente do ensino remoto, haja vista a falta do laboratório de eletrônica analógica. Embora alguns alunos (poucos) da turma não tivessem acesso ao computador, o trabalho era em equipe de 4 pessoas, sendo possível ainda realizar 0 projeto. O uso do programa possibilitou a equipe ter uma visão bem melhor do trabalho proposto, pois a plotagem no tempo das formas de onda de saída dos blocos funcionais foi prática e funcional. Somado a isso, possuía configuração de visualização da FFT (para avaliar a resposta em frequência das saídas dos blocos funcionais) simples e direta, não necessitando do aluno fazer um código computacional ou declarar equações para o cálculo.

O entendimento do bloco do filtro passa-faixa foi facilitado pelo uso do software, pois foi possível gerar a FFT rapidamente e avaliar a distribuição do espectro de frequência, no qual o grupo pôde verificar se a filtragem estava sendo correta ou incorretamente implementada. Um outro fator importante foi o projeto do amplificador pois existem várias configurações possíveis para sua construção, além de poder ser composto por estágio único ou múltiplo. Para o projeto, esse bloco deveria ser feito apenas com o conhecimento do ganho de amplificação. Como deveria ser utilizado o TBJ, constatou-se que o software possuía uma extensa lista de tipos de transistores, com várias especificações presentes; isso foi uma vantagem. O cálculo do THD também foi feito de modo compacto e direto, não necessitando definir as equações matemáticas.

Professores das áreas de engenharias ou similares devem avaliar a aplicação da metodologia ativa de uso de algum software adequado para melhorar o ensino de engenharia. Por exemplo, o mesmo programa utilizado (Ltspice) poderia ser destinado a 
matérias como: circuitos elétricos, processamento digital de sinais, teoria da comunicação e eletrônica de potência, além da própria eletrônica analógica.

\section{CONCLUSÃO}

O trabalho objetivou ser um relato de experiência do projeto de distorção harmônica total (THD), apresentado na disciplina de eletrônica analógica 2 da Universidade Federal do Pará (UFPA). O projeto consistiu em gerar uma forma de onda senoidal com baixo THD, a partir de 4 blocos funcionais: Gerador de onda quadrada com CI 555, filtro, multiplicador de frequência e amplificador com TBJ. O grupo conseguiu realizar o projeto no software Ltspice e inclusive o recomenda por ser bem prático de utilizar. Obteve-se THD (pelo software) de 0,76\% na saída do multiplicador de frequência testado isoladamente; $6,74 \%$ na saída do amplificador também testado separado e 5,55\% na saída do circuito geral, com todos os blocos juntos. Como foi especificado obter um valor menor que 10\%, conseguiu-se atender as expectativas. Além disso, a saída na carga de $2 \mathrm{~K} \Omega$ teve tensão pico-a-pico de $16,6 \mathrm{~V}$, que se adequou ao pedido entre 15 $20 \mathrm{~V}$.

A experiência da equipe foi muito produtiva e houve grande aprendizado de conhecimentos importantes a serem utilizados no decorrer do curso e nas futuras práticas profissionais. Devido a pandemia da COVID-19 e a não possibilidade de aulas laboratoriais, o uso de software de simulação na disciplina de eletrônica analógica 2 foi fundamental para reduzir as lacunas existentes pela falta da prática. Espera-se que o uso de programas computacionais possa ser aplicado a inúmeras outras disciplinas do curso de graduação em engenharia elétrica, ou de outras áreas da engenharia, principalmente as que precisam de laboratórios. Dessa forma, os alunos terão a possibilidade de compreender de modo melhor os conceitos teóricos abordados nas aulas, adquirindo experiências de simulação e quando as aulas presenciais voltarem terão noções do que fazer na prática.

\section{REFERÊNCIAS}

BERNARDO, J. Filtro Passivo Passa Faixa ou Passa Banda. Disponível em: https://eletronworld.com.br/eletronica/filtro-passivo-passa-faixa-ou-passa-banda/. Acesso em: 23 abr, 2021.

BOYLESTAD, R. L; NASHELSKY, L. Dispositivos Eletrônicos e Teoria de Circuitos. 11.ed. São Paulo: Person Education do Brasil, 2013.

BRAGA, N. C. O Circuito Integrado 555 Mágico. 1a. ed. São Paulo: Editora Newton C. Braga, 2016.

FERREIRA, D. H. L; BRANCHI, B. A; SUGAHARA, C. R. Processo de Ensino e Aprendizagem no Contexto das Aulas e Atividades Remotas no Ensino Superior em Tempo de Pandemia Covid-19. Revista Práxis, v.12, n.1 (Sup). ISSN online: 2176 9230. ISSN impresso: 1984 - 4239. 2020.

FREIRE, P. Pedagogia da autonomia. São Paulo: Paz e terra, 1996.

LIMA JUNIOR, A. W. Eletricidade \& Eletronica Básica. 3ª . ed. Rio de Janeiro 
MUSSOI, F. L. R. Resposta em Frequência - FILTROS PASSIVOS. Florianópolis: Centro Federal de Educação Tecnológica de Santa Catarina, 2004. Disponível em: https://intranet.ctism.ufsm.br/gsec/Apostilas/filtropassivo.pdf. Acesso em: 09 mai, 2021.

RIBEIRO, L. R. C. Aprendizagem Baseada em Problemas (PBL) na Educação em Engenharia. Revista do Ensino de Engenharia, v.27, n.2, p.23-32, 2008 - ISSN 0101 5001.

ROSU, L. Frequency Multipliers: There are few approaches how to generate a high frequency signal for microwaves frequencies. Disponível em:

$<$ https://doc.xdevs.com/docs/_Materials/Frequency_multiplier_circuits_survey_and_theory ROSU.pdf>. Acesso em: 23, abr. 2021.

SCHERZ, P; MONK, S. Título: Practical Eletronics for Inventors. Third Edition. 2013.

SEDRA, A; SMITH, K. Microeletronic Circuits. 7 .ed. New York: Oxford University Press, p. 209, c2015.

SILVA, F.S.S. da.; MORAIS, L.J.O.; CUNHA, I.P.R. (2011). Dificuldades dos professores de Biologia em ministrar aulas práticas em escolas públicas e privadas do município de Imperatriz (MA). Revista UNI, Imperatriz, MA, n. 1, p. 135-149. Starlin Alta Con. Com. Ltda, 2009.

\title{
EXPERIENCE REPORT ON THE TOTAL HARMONIC DISTORTION PROJECT: ACTIVE METHODOLOGY IN ANALOGUE ELECTRONICS IN THE COVID-19 PANDEMIC CONTEXT
}

\begin{abstract}
Due to the large number of subjects covered, students of courses in fields of applied sciences, such as electrical engineering, commonly have difficulties in assimilating the most important topics. Among the facilitating resources are laboratory classes, where the student can confront and experience theoretical concepts in practice. Currently, classes are being held remotely due to the COVID-19 pandemic. This imposes a great challenge to the quality of the training of students. A viable alternative and mainly adequate to the current reality is the use of computer simulators. In disciplines such as analog electronics, simulators can be used as a tool for active methodology, enabling the consolidation of knowledge in a practical, well-structured way that encourages the creativity of students, as if they were in a real laboratory. The present work aims to report the experience in the execution of an educational project involving the concept of Total Harmonic Distortion (THD) in electronic circuits, during the analogical discipline 2, of the Electrical Engineering course at the Federal University of Pará (UFPA). In particular, it seeks to evaluate the importance of using simulation software as an active methodology tool in times of restricted social contact, as is the case currently experienced.
\end{abstract}

Keywords: Amplifiers, Total Harmonic Distortion, Analog Electronics, Filters, Teaching Methodology. 